\title{
Homotopy sequence of a topological groupoid with a basegroup and an obstruction to presentability of proper regular Lie groupoids
}

\author{
B. Jelenc $\cdot$ J. Mrčun
}

Received: 6 March 2013 / Accepted: 25 November 2013 / Published online: 13 December 2013

(C) Tbilisi Centre for Mathematical Sciences 2013

\begin{abstract}
A topological groupoid $\mathscr{G}$ is $K$-pointed, if it is equipped with a homomorphism from a topological group $K$ to $\mathscr{G}$. We describe the homotopy groups of such $K$-pointed topological groupoids and relate these groups to the ordinary homotopy groups in terms of a long exact sequence. As an application, we give an obstruction to presentability of proper regular Lie groupoids.
\end{abstract}

Keywords Topological groupoids $\cdot$ Morita category $\cdot$ Homotopy groups $\cdot$ Serre fibrations

Mathematics Subject Classification (1991) 22A22 $\quad$ 55Q05 $58 \mathrm{H} 05$

\section{Introduction}

Topological and Lie groupoids are geometric objects that can be used to represent various singular geometric structures. Examples of such structures include foliations, equivalence relations, group actions, orbifolds, etc. The topological groupoids associated to these geometric structures are usually determined up to Morita equivalence.

Communicated by Ross Street.

This work was supported in part by the Slovenian Research Agency (ARRS) project J1-2247.

B. Jelenc

Institute of Mathematics, Physics and Mechanics, University of Ljubljana,

Jadranska 19, 1000 Ljubljana, Slovenia

e-mail: blaz.jelenc@imfm.si

J. Mrčun $(\varangle)$

Department of Mathematics, University of Ljubljana, Jadranska 19,

1000 Ljubljana, Slovenia

e-mail: janez.mrcun@fmf.uni-lj.si 
Homotopy groups are examples of Morita invariants of topological groupoids. They can be defined as the homotopy groups of the associated classifying space (see e.g. $[6,13])$. An equivalent description of these groups is given by the group of homotopy classes of principal $(\mathscr{G}, a)$-bundles over $\left(I^{n}, \partial I^{n}\right)[6,10,18]$. Here $(\mathscr{G}, a)$ is a pointed topological groupoid with basepoint $a$ in the space of objects $\mathscr{G}_{0}$ of $\mathscr{G}$. This approach has several advantages, for instance, it enables us to describe the notion of a Serre fibration in the Morita category of topological groupoids and derive the associated long exact sequence of homotopy groups ([10], see also [19] for a study of fibrations in the context of stacks).

The description of homotopy groups in terms of principal bundles over $\left(I^{n}, \partial I^{n}\right)$ also allows us to consider the homotopy groups of a topological groupoid with a more general base instead of a simple basepoint in its space of objects. A homomorphism $\rho$ from a locally compact Hausdorff topological group $K$ to a topological groupoid $\mathscr{G}$ defines a $K$-basegroup in $\mathscr{G}$ at a point $a$ in $\mathscr{G}_{0}$. We say that such a groupoid with a $K$ basegroup is $K$-pointed and denote it by the triple $(\mathscr{G}, a, \rho)$. The $K$-pointed topological groupoids form a Morita category, where morphisms are given by the (isomorphism classes of) principal bundles. Such a principal $(\mathscr{G}, a, \rho)$-bundle over $(\mathscr{H}, b, v)$ is a pointed space $(P, p)$ which is a principal $\mathscr{G}$-bundle over $\mathscr{H}[7,17,18]$ compatible with the action of the basegroups. We can extend this Morita category further to include $K$-marked topological groupoids, which are triples $(\mathscr{G}, A, \rho)$, where $A$ is a subset of $\mathscr{G}_{0}$ and $\rho$ is a continuous choice of a $K$-basegroup at each $a \in A$. For instance, this Morita category of $K$-marked topological groupoids includes $\left(K \times I^{n}, \partial I^{n}, \tau\right)$, where $\tau$ is the inclusion. We define the $n$-th homotopy group of a $K$-pointed topological groupoid $(\mathscr{G}, a, \rho)$ as the group of homotopy classes of principal $(\mathscr{G}, a, \rho)$-bundles over $\left(K \times I^{n}, \partial I^{n}, \tau\right)$, and denote it by $\pi_{n}^{K}(\mathscr{G})=\pi_{n}^{K}(\mathscr{G}, a, \rho)$. It is clear that this gives us a more refined version of homotopy groups, as the elements of $\pi_{n}^{K}(\mathscr{G})$ can be seen as elements of the ordinary homotopy group $\pi_{n}(\mathscr{G})=\pi_{n}(\mathscr{G}, a)$ with an additional left $K$-action. Similar constructions are known in the context of orbifolds [2] and orbispaces [9].

Next, we associate to every topological groupoid $\mathscr{G}$ the groupoid $\mathscr{G}^{K}$, which is the action groupoid associated to the adjoint $\mathscr{G}$-action on the space of all morphisms from $K$ to $\mathscr{G}$. This groupoid plays the role of the exponential in the Morita category of topological groupoids. This exponential provides an alternative description of the homotopy groups of a $K$-pointed topological groupoid $(\mathscr{G}, a, \rho)$ as ordinary homotopy groups of the pointed groupoid $\left(\mathscr{G}^{K}, \rho\right)$. We also extend the notion of a Serre fibration to the notion of a Serre $K$-fibration. We are especially interested in the canonical functor $\omega: \mathscr{G}^{K} \rightarrow \mathscr{G}$ that gives the relation between homotopy groups of a $K$ pointed groupoid $(\mathscr{G}, a, \rho)$ and ordinary homotopy groups of $(\mathscr{G}, a)$. When $\omega$ is a Serre fibration, this relation is expressed in terms of a long exact sequence

$$
\cdots \rightarrow \pi_{n}\left(\operatorname{hom}\left(K, \mathscr{G}_{a}\right)\right) \rightarrow \pi_{n}^{K}(\mathscr{G}) \rightarrow \pi_{n}(\mathscr{G}) \rightarrow \pi_{n-1}\left(\operatorname{hom}\left(K, \mathscr{G}_{a}\right)\right) \rightarrow \cdots
$$

where $\operatorname{hom}\left(K, \mathscr{G}_{a}\right)$ is the space of all homomorphisms from $K$ to the isotropy group $\mathscr{G}_{a}$ of $\mathscr{G}$ at $a$.

In the last part of the paper, we study the homotopy groups of $K$-pointed connected proper regular Lie groupoids. Choosing the $K$-basegroup of such a groupoid to be 
given by the Lie group $\mathrm{K}_{a}(\mathscr{G})$ of its ineffective arrows at $a \in \mathscr{G}_{0}$, the long exact sequence associated to the functor $\omega$ gives us the monodromy map

$$
\partial: \pi_{1}(\mathscr{G}) \rightarrow \pi_{0}\left(\operatorname{Aut}\left(\mathrm{K}_{a}(\mathscr{G})\right)\right)
$$

which is a Morita invariant of $\mathscr{G}$. This map provides us with an obstruction to presentability of connected proper regular Lie groupoids as global quotients (see Theorem 15). We show that if such a groupoid $\mathscr{G}$ is Morita equivalent to the action groupoid associated to an action of a connected compact Lie group on a smooth manifold, then there exists a faithful unitary representation of $\mathrm{K}_{a}(\mathscr{G})$ such that the corresponding character is fixed under precomposition with any automorphism representing a class in the image of the monodromy map $\partial: \pi_{1}(\mathscr{G}) \rightarrow \pi_{0}\left(\operatorname{Aut}\left(\mathrm{K}_{a}(\mathscr{G})\right)\right)$.

\section{Morita category of group-marked topological groupoids}

\subsection{Topological groupoids}

To fix the notations, let us first recall some basic definitions and facts (for details and examples, see e.g. $[12,16,17])$. A groupoid is a small category in which all arrows are invertible. In particular, a groupoid $\mathscr{G}$ has its set of objects $\mathscr{G}_{0}$ and its set of arrows $\mathscr{G}_{1}$. Each arrow $g$ of $\mathscr{G}$ has its source object $\mathrm{s}(g)$ and its target object $\mathrm{t}(g)$. For two objects $x, x^{\prime} \in \mathscr{G}_{0}$ we denote by $\mathscr{G}\left(x, x^{\prime}\right)=\mathrm{s}^{-1}(x) \cap \mathrm{t}^{-1}\left(x^{\prime}\right)$ the set of arrows from $x$ to $x^{\prime}$ in $\mathscr{G}$. The product arrow $g g^{\prime} \in \mathscr{G}_{1}$ of two arrows $g, g^{\prime} \in \mathscr{G}_{1}$ is defined if $\mathrm{s}(g)=\mathrm{t}\left(g^{\prime}\right)$, and in this case $\mathrm{s}\left(g g^{\prime}\right)=\mathrm{s}\left(g^{\prime}\right)$ and $\mathrm{t}\left(g g^{\prime}\right)=\mathrm{t}(g)$. Each object $x$ has its unit arrow $1_{x} \in \mathscr{G}(x, x)$, and each arrow $g$ has its inverse arrow $g^{-1}$. A groupoid $\mathscr{G}$ is sometimes denoted by $\left(\mathscr{G}_{1} \rightrightarrows \mathscr{G}_{0}\right)$. The set $\mathscr{G}(x, x)$ is a group, called the isotropy group of $\mathscr{G}$ at the object $x$, and denoted also by $\mathscr{G}_{x}$ or $\mathrm{I}_{x}(\mathscr{G})$.

A topological groupoid is a groupoid $\mathscr{G}$ with topology on $\mathscr{G}_{0}$ and on $\mathscr{G}_{1}$ such that all the structure maps mentioned above are continuous. In particular, the isotropy groups of a topological groupoid are topological groups. A homomorphism of topological groupoids is a continuous functor between them. In this way we get the category of topological groupoids, which we denote by Gpd.

First examples of topological groupoids are topological spaces and topological groups: any topological space $X$ can be viewed as the (unit) topological groupoid ( $X \rightrightarrows X)$, and any topological group $G$ is a topological groupoid with only one object $(G \rightrightarrows *)$. The category of topological spaces and the category of topological groups are both full subcategories of Gpd.

\subsection{Actions of topological groupoids}

Recall that a right action of a topological groupoid $\mathscr{G}$ on a topological space $X$ along a (continuous) map $\epsilon: X \rightarrow \mathscr{G}_{0}$ is a map $\mu: X \times \mathscr{G}_{0} \mathscr{G}_{1} \rightarrow X,(x, g) \mapsto x g$, which is defined for each pair $(x, g) \in X \times \mathscr{G}_{1}$ with $\epsilon(x)=\mathrm{t}(g)$ and satisfies the usual properties of an action. For such a right $\mathscr{G}$-action we write $x \mathscr{G}=\mu\left(x, \mathrm{t}^{-1}(x)\right)$ for the orbit through $x$ and $X / \mathscr{G}$ for the associated space of orbits. We have the associated action groupoid $X \rtimes \mathscr{G}=\left(X \times \mathscr{G}_{0} \mathscr{G}_{1} \rightrightarrows X\right)$, which is the topological groupoid in which the source map is the action $\mu$, the target map is the first projection and the 
product is given by $(x, g)\left(x^{\prime}, g^{\prime}\right)=\left(x, g g^{\prime}\right)$. Analogously, one defines a left action of a topological groupoid $\mathscr{H}$ on $X$ and the associated action groupoid $\mathscr{H} \ltimes X$.

\subsection{Morita category of topological groupoids}

Let $\mathscr{G}$ and $\mathscr{H}$ be topological groupoids. A principal $\mathscr{G}$-bundle over $\mathscr{H}$ is a space $P$, equipped with a left $\mathscr{H}$-action along $\pi: P \rightarrow \mathscr{H}_{0}$ and a right $\mathscr{G}$-action along $\epsilon: P \rightarrow \mathscr{G}_{0}$ such that

(i) the map $\pi$ has local sections (i.e. for any $y \in \mathscr{H}_{0}$ there exist a section of $\pi$, defined on an open neighbourhood of $y$ ),

(ii) for every $h \in \mathscr{H}_{1}, p \in P$ and $g \in \mathscr{G}_{1}$ with $s(h)=\pi(p)$ and $\epsilon(p)=t(g)$ we have $\pi(p g)=\pi(p), \epsilon(h p)=\epsilon(p)$ and $h(p g)=(h p) g$, and

(iii) the map $P \times \mathscr{G}_{0} \mathscr{G}_{1} \rightarrow P \times \mathscr{H}_{0} P,(p, g) \mapsto(p, p g)$, is a homeomorphism.

A morphism between principal $\mathscr{G}$-bundles over $\mathscr{H}$ is an $\mathscr{H}$ - $\mathscr{G}$-equivariant map between them. Any such homomorphism is in fact an isomorphism. We want to point out that $\mathscr{G}$ itself is a principal $\mathscr{G}$-bundle over $\mathscr{G}$, where the condition (i) is verified by the unit map $\mathscr{G}_{0} \rightarrow \mathscr{G}$, which is a global section of the target map. Condition (i) assures that principal bundles behave well locally, and this is essential for the results of the first two sections of this paper. Note, however, that in the context of Lie groupoids we consider smooth principal bundles, where condition (i) follows from a stronger assumption that the map $\pi$ is a surjective submersion.

For a principal $\mathscr{G}$-bundle $P$ over $\mathscr{H}$ and a principal $\mathscr{H}$-bundle $Q$ over $\mathscr{K}$ we have the tensor product $Q \otimes P=Q \otimes \mathscr{H} P$, which is a principal $\mathscr{G}$-bundle over $\mathscr{K}$. The space $Q \otimes P$ is the space of orbits of the diagonal $\mathscr{H}$-action on the fibered product $Q \times \mathscr{H}_{0} P[17,18]$. The Morita category GPD of topological groupoids is the category with topological groupoids as objects and isomorphism classes of principal bundles as morphisms, and with composition induced by the tensor product. Morita equivalences are the isomorphisms in GPD. The category of topological spaces is a full subcategory of the Morita category GPD. There is a natural functor $\langle-\rangle:$ Gpd $\rightarrow$ GPD, which is identity on objects, while it maps a homomorphism $\phi: \mathscr{H} \rightarrow \mathscr{G}$ to the (isomorphism class of the) principal $\mathscr{G}$-bundle $\langle\phi\rangle=\mathscr{H}_{0} \times \mathscr{G}_{0} \mathscr{G}_{1}$ over $\mathscr{H}$. We also have the Morita bicategory of topological groupoids in which principal bundles are 1-morphisms and morphisms of principal bundles are 2-morphisms.

\subsection{Pointed and marked topological groupoids}

A pointed topological groupoid is a topological groupoid $\mathscr{G}$, together with a basepoint $a \in \mathscr{G}_{0}$ (see [8,18]). More generally, a marked topological groupoid is a pair $(\mathscr{G}, A)$, where $\mathscr{G}$ is a topological groupoid and $A$ is a subset of $\mathscr{G}_{0}$. A pointed topological groupoid $(\mathscr{G}, a)$ can therefore be seen as the marked topological groupoid $(\mathscr{G},\{a\})$. A homomorphism between marked topological groupoids $\phi:(\mathscr{H}, B) \rightarrow(\mathscr{G}, A)$ is a homomorphism $\phi: \mathscr{H} \rightarrow \mathscr{G}$ such that $\phi(B) \subset A$. A principal $(\mathscr{G}, A)$-bundle over $(\mathscr{H}, B)$ is a pair $(P, \sigma)$, where $P$ is a principal $\mathscr{G}$-bundle over $\mathscr{H}$ and $\sigma: B \rightarrow P$ is a section of $\pi: P \rightarrow \mathscr{H}_{0}$ such that $\epsilon:(P, \sigma(B)) \rightarrow\left(\mathscr{G}_{0}, A\right)$ is a map of topo- 
logical pairs and $\pi:(P, \sigma(B)) \rightarrow\left(\mathscr{H}_{0}, B\right)$ is a map of topological pairs with local sections (i.e. for any $b \in B$ there exist a section of $\pi$, defined on an open neighbourhood $V$ of $b$ in $\mathscr{H}_{0}$, which is a map of topological pairs $\left.(V, V \cap B) \rightarrow(P, \sigma(B))\right)$. In particular, if $(\mathscr{G}, a)$ and $(\mathscr{H}, b)$ are pointed topological groupoids and $(P, \sigma)$ is principal $(\mathscr{G}, a)$-bundle over $(\mathscr{H}, b)$, then we write $(P, \sigma)=(P, \sigma(b))$. Morphisms between principal $(\mathscr{G}, A)$-bundles over $(\mathscr{H}, B)$ are morphisms of principal $\mathscr{G}$-bundles over $\mathscr{H}$ which commute the given sections. One can check that the tensor product of principal bundles with sections has a natural section as well, thus we obtain the Morita category GPD $_{[*]}$ of marked topological groupoids. As a full subcategory of $\mathrm{GPD}_{[*]}$, we have the Morita category $\mathrm{GPD}_{*}$ of pointed topological groupoids.

\subsection{Group-marked topological groupoids}

Let $K$ be a fixed locally compact Hausdorff topological group. A $K$-basegroup in a topological groupoid $\mathscr{G}$ is a homomorphism $\varphi: K \rightarrow \mathscr{G}$, where we view $K$ as the topological groupoid ( $K \rightrightarrows *$ ). In particular, such a $K$-basegroup is a homomorphism of topological groups $\varphi: K \rightarrow \mathscr{G}_{\varphi(*)}$. We write

$$
\operatorname{hom}(K, \mathscr{G})=\operatorname{Gpd}((K \rightrightarrows *), \mathscr{G})
$$

for the space of all $K$-basegroups of $\mathscr{G}$, equipped with the compact-open topology, and $\omega: \operatorname{hom}(K, \mathscr{G}) \rightarrow \mathscr{G}_{0}$ for the natural map given by $\omega(\varphi)=\varphi(*)$. Any homomorphism $\phi \in \operatorname{Gpd}(\mathscr{H}, \mathscr{G})$ induces a map $\phi_{*}: \operatorname{hom}(K, \mathscr{H}) \rightarrow \operatorname{hom}(K, \mathscr{G})$. A $K$-pointed topological groupoid is a topological groupoid $\mathscr{G}$ together with a $K$-basegroup $\rho$ in $\mathscr{G}$; we denote such a $K$-pointed topological groupoid by $(\mathscr{G}, a, \rho)$, where $a=\rho(*)$. A homomorphism between $K$-pointed topological groupoids $(\mathscr{H}, b, v)$ and $(\mathscr{G}, a, \rho)$ is a homomorphism $\phi \in \operatorname{Gpd}(\mathscr{H}, \mathscr{G})$ with $\phi_{*}(v)=\rho$. In this way we obtain the category $\operatorname{Gpd}_{K}$ of $K$-pointed topological groupoids, which is in other words the coslice category $(K \rightrightarrows *) \downarrow$ Gpd.

For a principal $\mathscr{G}$-bundle $P$ over $\mathscr{H}$, any point $p \in P$ induces a homomorphism of groups $P_{p}: \mathscr{H}_{\pi(p)} \rightarrow \mathscr{G}_{\epsilon(p)}$, uniquely determined by the property that $h p=p P_{p}(h)$ for any $h \in \mathscr{H}_{\pi(p)}$. A principal $(\mathscr{G}, a, \rho)$-bundle over $(\mathscr{H}, b, v)$ is a principal $(\mathscr{G}, a)$ bundle $(P, p)$ over $(\mathscr{H}, b)$ satisfying $P_{p} \circ v=\rho$. We obtain the Morita category $\mathrm{GPD}_{K}$ of $K$-pointed topological groupoids, where the morphisms are given by the isomorphism classes of principal bundles.

More generally, a $K$-marked topological groupoid is a triple $(\mathscr{G}, A, \rho)$, where $A \subset \mathscr{G}_{0}$ and $\rho: K \times A \rightarrow \mathscr{G}$ is a continuous functor which is identity on objects. A principal $(\mathscr{G}, A, \rho)$-bundle over $(\mathscr{H}, B, v)$ is a principal $(\mathscr{G}, A)$-bundle $(P, \sigma)$ over $(\mathscr{H}, B)$ satisfying $P_{\sigma(b)}(v(k, b))=\rho(k, \epsilon(\sigma(b)))$ for any $b \in B$ and $k \in K$. Again we obtain the Morita category $\operatorname{GPD}_{[K]}$ of $K$-marked topological groupoids, where the morphisms are given by isomorphism classes of principal bundles. The category $\mathrm{GPD}_{K}$ is a full subcategory of $\mathrm{GPD}_{[K]}$. An example of a $K$-marked topological groupoid is $\left(K \times I^{n}, \partial I^{n}, \tau\right)$, where $\tau: K \times \partial I^{n} \rightarrow K \times I^{n}$ is the inclusion. 


\subsection{Homotopy}

Let $(\mathscr{G}, A, \rho),(\mathscr{H}, B, v)$ be $K$-marked topological groupoids and $(P, \sigma),\left(P^{\prime}, \sigma^{\prime}\right)$ principal $(\mathscr{G}, A, \rho)$-bundles over $(\mathscr{H}, B, v)$. A homotopy from $(P, \sigma)$ to $\left(P^{\prime}, \sigma^{\prime}\right)$ is principal $(\mathscr{G}, A, \rho)$-bundle $(H, \lambda)$ over $(\mathscr{H} \times I, B \times I, v \times I)$ such that the restrictions of $(H, \lambda)$ to $(\mathscr{H} \times\{0\}, B \times\{0\}, v \times\{0\})$ and $(\mathscr{H} \times\{1\}, B \times\{1\}, v \times\{1\})$ are isomorphic to $(P, \sigma)$ respectively $\left(P^{\prime}, \sigma^{\prime}\right)$. We say that $(P, \sigma),\left(P^{\prime}, \sigma^{\prime}\right)$ are homotopic if such a homotopy exists. One may show that homotopy is an equivalence relation on the set of Morita morphisms from $(\mathscr{H}, B, v)$ to $(\mathscr{G}, A, \rho)$ and that this equivalence relation behaves well with respect to the composition in $\operatorname{GPD}_{[K]}$. (To show the transitivity of the relation, one needs to concatenate two homotopies, and this should be done by inserting a trivial homotopy in the middle to ensure that the concatenated principal bundle is locally trivial.) We denote by

$$
\operatorname{hGPD}_{[K]}((\mathscr{H}, B, v),(\mathscr{G}, A, \rho))=[(\mathscr{H}, B, v),(\mathscr{G}, A, \rho)]
$$

the set of homotopy classes of principal $(\mathscr{G}, A, \rho)$-bundles over $(\mathscr{H}, B, v)$. We obtain the homotopy Morita category hGPD $[K]$ of $K$-marked topological groupoids.

\subsection{Homotopy groups}

We define the $n$-th homotopy group of a $K$-pointed topological groupoid $(\mathscr{G}, a, \rho)$ by

$$
\pi_{n}^{K}(\mathscr{G})=\pi_{n}^{K}(\mathscr{G}, a, \rho)=\left[\left(K \times I^{n}, \partial I^{n}, \tau\right),(\mathscr{G}, a, \rho)\right] .
$$

As usual, this is just a pointed set for $n=0$, a group for $n=1$ and an abelian group for $n \geq 2$. For $K=1$ we recover the usual homotopy groups $\pi_{n}(\mathscr{G}, a)$ of the pointed topological groupoid $(\mathscr{G}, a)$. By definition we see that $\pi_{n}^{K}$ is homotopy Morita invariant, as it is a functor defined on the category $\mathrm{GPD}_{K}$ and also on $\mathrm{hGPD}_{K}$. Any homomorphism $K \rightarrow K^{\prime}$ between locally compact Hausdorff topological groups induces a natural transformation from $\pi_{n}^{K^{\prime}}$ and $\pi_{n}^{K}$ over the natural functor GPD $K^{\prime} \rightarrow$ $\mathrm{GPD}_{K}$.

\section{Homotopy sequence of a topological groupoid with a basegroup}

\subsection{The groupoid $\mathscr{G}^{K}$}

Let $K$ be a locally compact Hausdorff topological group. For a topological groupoid $\mathscr{G}$ we have the natural map $\omega: \operatorname{hom}(K, \mathscr{G}) \rightarrow \mathscr{G}_{0}, \varphi \mapsto \varphi(*)$. Now observe that there is a natural right (adjoint) $\mathscr{G}$-action on $\operatorname{hom}(K, \mathscr{G})$ along $\omega$, given by

$$
(\varphi g)(k)=g^{-1} \varphi(k) g=\left(\operatorname{Ad}_{g^{-1}} \circ \varphi\right)(k)
$$

for any $k \in K$. We denote by

$$
\mathscr{G}^{K}=\operatorname{hom}(K, \mathscr{G}) \rtimes \mathscr{G}
$$


the associated action groupoid. In particular, the map $\omega$ induces a homomorphism $\omega=\omega \mathscr{G}: \mathscr{G}^{K} \rightarrow \mathscr{G}$. The definition of $\mathscr{G}^{K}$ can be extended to a functor. Indeed, if $P$ is a principal $\mathscr{G}$-bundle over $\mathscr{H}$, we take

$$
P^{K}=\operatorname{hom}(K, \mathscr{H}) \times \mathscr{H}_{0} P .
$$

The space $P^{K}$ has a natural structure of a principal $\mathscr{G}^{K}$-bundle over $\mathscr{H}^{K}$, given by maps $\pi(\varphi, p)=\varphi, \epsilon(\varphi, p)=P_{p} \circ \varphi$ and actions $\left(\operatorname{Ad}_{h} \circ \varphi, h\right)(\varphi, p)=\left(\operatorname{Ad}_{h} \circ \varphi, h p\right)$, $(\varphi, p)\left(P_{p} \circ \varphi, g\right)=(\varphi, p g)$. One can check that this gives us a functor $(-)^{K}$ : GPD $\rightarrow$ GPD. Note that we have a natural isomorphism between $P^{K} \otimes\langle\omega \mathscr{G}\rangle$ and $\langle\omega \mathscr{H}\rangle \otimes P$. Note also that a homomorphism $\phi: \mathscr{H} \rightarrow \mathscr{G}$ induces a homomorphism $\phi^{K}: \mathscr{H}^{K} \rightarrow \mathscr{G}^{K}$ in the obvious way and that $\left\langle\phi^{K}\right\rangle$ is naturally isomorphic to $\langle\phi\rangle^{K}$.

Proposition 1 Let $\mathscr{G}, \mathscr{H}$ be topological groupoids and $K$ a locally compact Hausdorff topological group. There is a natural bijection

$$
\operatorname{GPD}(K \times \mathscr{H}, \mathscr{G}) \cong \operatorname{GPD}\left(\mathscr{H}, \mathscr{G}^{K}\right) .
$$

Proof Let $P$ be a principal $\mathscr{G}$-bundle over $K \times \mathscr{H}$. For any $p \in P$ we have the associated homomorphism of topological groups $P_{p}: K \times \mathscr{H}_{\pi(p)} \rightarrow \mathscr{G}_{\epsilon(p)}$. There is also the associated map $\bar{\epsilon}: P \rightarrow \operatorname{hom}(K, \mathscr{G})$ given by $\bar{\epsilon}(p)(k)=P_{p}\left(k, 1_{\pi(p)}\right)$. Explicitly, the map $\bar{\epsilon}$ is characterized by the equality

$$
\left(k, 1_{\pi(p)}\right) p=p \bar{\epsilon}(p)(k),
$$

which holds for any $k \in K$. One can check that $\bar{\epsilon}$ is $\mathscr{G}$-equivariant, so we have a right $\mathscr{G}^{K}$-action on $P$ along $\bar{\epsilon}$. Furthermore, this action commutes with the left $\mathscr{H}$ action and $P$ is also a principal $\mathscr{G}^{K}$-bundle over $\mathscr{H}$. Finally, observe that $\epsilon$ can be reconstructed from $\bar{\epsilon}$ as $\epsilon=\omega \circ \bar{\epsilon}$, and the left $K$-action on $P$ can be reconstructed by Eq. (1).

Remark In fact we proved that we have a natural isomorphism of groupoids

$$
\underline{\operatorname{GPD}}(K \times \mathscr{H}, \mathscr{G}) \cong \underline{\operatorname{GPD}}\left(\mathscr{H}, \mathscr{G}^{K}\right),
$$

where we use the underlined notation GPD to denote the set of principal bundles rather than the set of their isomorphism classes, i.e. the 1-morphisms in the Morita bicategory. We can lift this to the context of $K$-marked groupoids. In particular, we obtain a natural isomorphism of groupoids

$$
\underline{\operatorname{GPD}}_{[K]}\left(\left(K \times I^{n}, \partial I^{n}, \tau\right),(\mathscr{G}, a, \rho)\right) \cong \underline{\operatorname{GPD}}_{[*]}\left(\left(I^{n}, \partial I^{n}\right),\left(\mathscr{G}^{K}, \rho\right)\right),
$$

for any $K$-pointed topological groupoid $(\mathscr{G}, a, \rho)$.

The proposition shows that the groupoid $\mathscr{G}^{K}$ behaves as the exponent in the Morita category. For a more general study of exponents in the context of topological stacks, see [1]. 
Corollary 2 Let $K$ be a locally compact Hausdorff topological group. For any $K$ pointed topological groupoid $(\mathscr{G}, a, \rho)$ there is a natural isomorphism

$$
\pi_{n}^{K}(\mathscr{G}, a, \rho) \cong \pi_{n}\left(\mathscr{G}^{K}, \rho\right) .
$$

\subsection{Serre $K$-fibrations}

Let $\mathscr{H}$ and $\mathscr{G}$ be topological groupoids and let $P$ be a principal $\mathscr{G}$-bundle over $\mathscr{H}$. Recall from [10] that $P$ is a Serre fibration if for any given principal $\mathscr{H}$-bundle $\alpha$ over $I^{n}$, principal $\mathscr{G}$-bundle $\beta$ over $I^{n+1}$ and isomorphism $\chi$ from $\alpha \otimes P$ to $\left.\beta\right|_{I^{n} \times\{0\}}$, there exist a principal $\mathscr{H}$-bundle $\bar{\alpha}$ over $I^{n+1}$, a principal $\mathscr{G}$-bundle $\bar{\beta}$ over $I^{n+1}$ and isomorphisms of principal bundles $\bar{\chi}: \bar{\alpha} \otimes P \rightarrow \bar{\beta}, \zeta:\left.\alpha \rightarrow \bar{\alpha}\right|_{I^{n} \times\{0\}}$ and $\xi: \beta \rightarrow \bar{\beta}$ such that $\bar{\chi} \circ(\zeta \otimes P)=\xi \circ \chi$. This notion is defined in the Morita bicategory of topological groupoids, however it is clearly a well-defined property of a Morita map in GPD. It is a generalization of the notion of Serre fibration in the category of topological spaces, and can be stated as the existence of the morphism $\bar{\alpha}$ in the commutative diagram

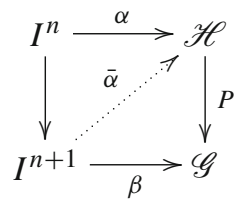

of 1-arrows in the Morita bicategory of topological groupoids. We say that a homomorphism $\phi: \mathscr{H} \rightarrow \mathscr{G}$ of topological groupoids is a Serre fibration if the associated principal $\mathscr{G}$-bundle $\langle\phi\rangle$ over $\mathscr{H}$ is a Serre fibration.

Let us state some basic properties of Serre fibrations which we will use in the rest of the paper (see [10], compare to [19]):

(i) Any Morita equivalence (i.e. an isomorphism in GPD) is a Serre fibration.

(ii) The composition of Serre fibrations in GPD is a Serre fibration.

(iii) If $\phi: \mathscr{H} \rightarrow \mathscr{G}$ is a homomorphism between topological groupoids such that $\phi: \mathscr{H}_{0} \rightarrow \mathscr{G}_{0}$ is a Serre fibration and $(\mathrm{t}, \phi): \mathscr{H}_{1} \rightarrow \mathscr{H}_{0} \times \mathscr{G}_{0} \mathscr{G}_{1}$ is a surjective Serre fibration, then $\phi$ is a Serre fibration.

(iv) Any principal $(\mathscr{G}, a)$-bundle $(P, p)$ over $(\mathscr{H}, b)$, which is a Serre fibration, induces a natural long exact sequence

$$
\begin{aligned}
\cdots & \rightarrow \pi_{n}\left(\mathscr{H} \ltimes \epsilon^{-1}(a)\right) \stackrel{\pi_{n}\left(\mathrm{pr}_{1}\right)}{\rightarrow} \pi_{n}(\mathscr{H}) \stackrel{\pi_{n}(P)}{\rightarrow} \pi_{n}(\mathscr{G}) \\
& \rightarrow \pi_{n-1}\left(\mathscr{H} \ltimes \epsilon^{-1}(a)\right) \rightarrow \cdots
\end{aligned}
$$

The action groupoid $\mathscr{H} \ltimes \epsilon^{-1}(a)$ is called the fiber of $P$ over $a$.

We say that a principal $\mathscr{G}$-bundle $P$ over $\mathscr{H}$ is a Serre $K$-fibration if the associated principal $\mathscr{G}^{K}$-bundle $P^{K}$ over $\mathscr{H}^{K}$ is a Serre fibration. By Proposition 1 we see that this is true if and only if for any given principal $\mathscr{H}$-bundle $\alpha$ over $K \times I^{n}$, principal 
$\mathscr{G}$-bundle $\beta$ over $K \times I^{n+1}$ and isomorphism $\chi$ from $\alpha \otimes P$ to $\left.\beta\right|_{K \times I^{n} \times\{0\}}$, there exist a principal $\mathscr{H}$-bundle $\bar{\alpha}$ over $K \times I^{n+1}$, a principal $\mathscr{G}$-bundle $\bar{\beta}$ over $K \times I^{n+1}$ and isomorphisms of principal bundles $\bar{\chi}: \bar{\alpha} \otimes P \rightarrow \bar{\beta}, \zeta:\left.\alpha \rightarrow \bar{\alpha}\right|_{K \times I^{n} \times\{0\}}$ and $\xi: \beta \rightarrow \bar{\beta}$ such that $\bar{\chi} \circ(\zeta \otimes P)=\xi \circ \chi$.

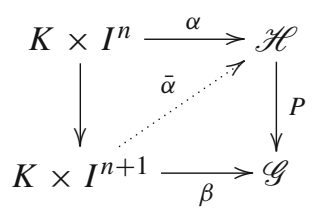

Analogously, we say that a homomorphism $\phi: \mathscr{H} \rightarrow \mathscr{G}$ is a Serre $K$-fibration if $\phi^{K}: \mathscr{H}^{K} \rightarrow \mathscr{G}^{K}$ is a Serre fibration, which is true of course if and only if $\langle\phi\rangle^{K}$ is a Serre fibration.

Lemma 3 Let $\phi: \mathscr{H} \rightarrow \mathscr{G}$ be a homomorphism of topological groupoids such that the map $(\mathrm{t}, \phi): \mathscr{H}_{1} \rightarrow \mathscr{H}_{0} \times \mathscr{G}_{0} \mathscr{G}_{1}$ is a surjective Serre fibration. Suppose that $X$ is a space with a right $\mathscr{G}$-action, $Y$ a space with a right $\mathscr{H}$-action and $f: Y \rightarrow X$ a $\phi$-equivariant Serre fibration. Then the induced homomorphism $Y \rtimes \mathscr{H} \rightarrow X \rtimes \mathscr{G}$ is a Serre fibration.

Proof We have to show that the map

$$
(\mathrm{t}, f \times \phi):(Y \rtimes \mathscr{H})_{1} \rightarrow(Y \rtimes \mathscr{H})_{0} \times_{(X \rtimes \mathscr{G})_{0}}(X \rtimes \mathscr{G})_{1}
$$

is a surjective Serre fibration. Equivalently, we have to show that the map $Y \times \mathscr{H}_{0} \mathscr{H}_{1} \rightarrow$ $Y \times \mathscr{H}_{0}\left(\mathscr{H}_{0} \times \mathscr{G}_{0} \mathscr{G}_{1}\right),(y, h) \mapsto(y, \mathrm{t}(h), \phi(h))$, is a surjective Serre fibration, which is true because it is a pull-back of the surjective Serre fibration $(\mathrm{t}, \phi): \mathscr{H}_{1} \rightarrow \mathscr{H}_{0} \times \mathscr{G}_{0} \mathscr{G}_{1}$.

Proposition 4 Let $K$ be a locally compact Hausdorff topological group and let $\phi$ : $\mathscr{H} \rightarrow \mathscr{G}$ be a homomorphism of topological groupoids such that the map $\phi_{*}:$ $\operatorname{hom}(K, \mathscr{H}) \rightarrow \operatorname{hom}(K, \mathscr{G})$ is a Serre fibration and the map $(\mathrm{t}, \phi): \mathscr{H}_{1} \rightarrow \mathscr{H}_{0} \times \mathscr{G}_{0}$ $\mathscr{G}_{1}$ is a surjective Serre fibration. Then $\phi$ is a Serre $K$-fibration.

Proof Note that the assumption that $\phi_{*}: \operatorname{hom}(K, \mathscr{H}) \rightarrow \operatorname{hom}(K, \mathscr{G})$ is a Serre fibration implies that $\phi: \mathscr{H}_{0} \rightarrow \mathscr{G}_{0}$ is also a Serre fibration. The proposition is hence a consequence of Lemma 3.

Proposition 5 Let $K$ be a locally compact Hausdorff topological group, let ( $\mathscr{G}, a, \rho)$, $(\mathscr{H}, b, v)$ be $K$-pointed topological groupoids and let $(P, p)$ be a principal $(\mathscr{G}, a, \rho)$ bundle over $(\mathscr{H}, b, v)$ which is a Serre $K$-fibration. We have a natural long exact sequence

$$
\begin{aligned}
\cdots & \rightarrow \pi_{n}\left(\mathscr{H}^{K} \ltimes \epsilon^{-1}(\rho),(v, p)\right) \rightarrow \pi_{n}^{K}(\mathscr{H}, b, v) \stackrel{\pi_{n}(P)}{\rightarrow} \pi_{n}^{K}(\mathscr{G}, a, \rho) \\
& \rightarrow \pi_{n-1}\left(\mathscr{H}^{K} \ltimes \epsilon^{-1}(\rho),(v, p)\right) \rightarrow \cdots
\end{aligned}
$$

Proof This is simply the long exact sequence of homotopy groups associated to the Serre fibration $P^{K}$. 
Proposition 6 Let $\mathscr{G}$ be a topological groupoid and $K$ a locally compact Hausdorff topological group. If $\omega: \operatorname{hom}(K, \mathscr{G}) \rightarrow \mathscr{G}_{0}$ is a Serre fibration, then $\omega: \mathscr{G}^{K} \rightarrow \mathscr{G}$ is a Serre fibration.

Proof This is a direct consequence of Lemma 3.

Theorem 7 Let $K$ be a locally compact Hausdorff topological group and $(\mathscr{G}, a, \rho) a$ $K$-pointed topological groupoid such that $\omega: \mathscr{G}^{K} \rightarrow \mathscr{G}$ is a Serre fibration. We have a natural long exact sequence

$$
\cdots \rightarrow \pi_{n}\left(\operatorname{hom}\left(K, \mathscr{G}_{a}\right)\right) \rightarrow \pi_{n}^{K}(\mathscr{G}) \stackrel{\pi_{n}(\omega)}{\rightarrow} \pi_{n}(\mathscr{G}) \stackrel{\partial}{\rightarrow} \pi_{n-1}\left(\operatorname{hom}\left(K, \mathscr{G}_{a}\right)\right) \rightarrow \cdots
$$

Proof The long exact sequence is the one associated to the Serre fibration $\omega: \mathscr{G}^{K} \rightarrow$ $\mathscr{G}$. This follows from Corollary 2 and the fact that the fiber of $\langle\omega\rangle$ over $a$ is Morita equivalent to the space $\operatorname{hom}\left(K, \mathscr{G}_{a}\right)$. Indeed, we have

$$
\langle\omega\rangle=\operatorname{hom}(K, \mathscr{G}) \times \mathscr{G}_{0} \mathscr{G}_{1},
$$

so the fiber of $\langle\omega\rangle$ over $a$ is the action groupoid associated to the $\mathscr{G}^{K}$-action on the space

$$
F=\operatorname{hom}(K, \mathscr{G}) \times \mathscr{G}_{0} \mathscr{G}(a,-)
$$

where $\mathscr{G}(a,-)=\mathrm{s}^{-1}(a)$ is the source-fiber over $a$ in $\mathscr{G}$. Now note that the $\mathscr{G}^{K}$-action on $F$ is free and the subspace $\operatorname{hom}(K, \mathscr{G}) \times \mathscr{G}_{0}\left\{1_{a}\right\} \cong \operatorname{hom}\left(K, \mathscr{G}_{a}\right)$ of $F$ intersects each orbit of $\mathscr{G}^{K}$-action in exactly one point. One can then see that the natural inclusion of the space $\operatorname{hom}\left(K, \mathscr{G}_{a}\right)$ into the fiber $\mathscr{G}^{K} \ltimes F$ gives a Morita equivalence.

It turns out that in concrete examples it is often too optimistic to expect that $\omega$ : $\mathscr{G}^{K} \rightarrow \mathscr{G}$ is a Serre fibration, but it may well happen that this homomorphism is a Serre fibration when restricted to a path-component of the topological groupoid $\mathscr{G}^{K}$. (We say that a topological groupoid $\mathscr{H}$ is path-connected if $\pi_{0}(\mathscr{H})=1$. A path-component of a topological groupoid $\mathscr{H}$ is a maximal path-connected (full) subgroupoid of $\mathscr{H}$. The space of objects of a path-component of $\mathscr{H}$ is a minimal $\mathscr{H}$-invariant union of path-components of the space $\mathscr{H}_{0}$.) Therefore, let us introduce the following notation: For a $K$-pointed path-connected topological groupoid $(\mathscr{G}, a, \rho)$, denote by $\mathscr{G}_{\rho}^{K}$ the path-component of $\mathscr{G}^{K}$ with $\rho \in\left(\mathscr{G}_{\rho}^{K}\right)_{0}$, and write hom $(K, \mathscr{G})_{\rho}=\left(\mathscr{G}_{\rho}^{K}\right)_{0}$. Denote also hom $(K, \mathscr{G})_{a, \rho}=\operatorname{hom}\left(K, \mathscr{G}_{a}\right) \cap \operatorname{hom}(K, \mathscr{G})_{\rho}$. Lemma 3 now implies:

Proposition 8 Let $K$ be a locally compact Hausdorff topological group and $(\mathscr{G}, a, \rho)$ a K-pointed path-connected topological groupoid. If $\omega: \operatorname{hom}(K, \mathscr{G})_{\rho} \rightarrow \mathscr{G}_{0}$ is a Serre fibration, then $\omega: \mathscr{G}_{\rho}^{K} \rightarrow \mathscr{G}$ is a Serre fibration.

Theorem 9 Let $K$ be a locally compact Hausdorff topological group and (G, a, $\rho)$ a K-pointed path-connected topological groupoid such that $\omega: \mathscr{G}_{\rho}^{K} \rightarrow \mathscr{G}$ is a Serre fibration. We have a natural long exact sequence 


$$
\begin{aligned}
& \cdots \rightarrow \pi_{n}\left(\operatorname{hom}\left(K, \mathscr{G}_{a}\right)\right) \rightarrow \pi_{n}^{K}(\mathscr{G}) \stackrel{\pi_{n}(\omega)}{\rightarrow} \pi_{n}(\mathscr{G}) \stackrel{\partial}{\rightarrow} \pi_{n-1}\left(\operatorname{hom}\left(K, \mathscr{G}_{a}\right)\right) \rightarrow \cdots \\
& \cdots \rightarrow \pi_{1}\left(\operatorname{hom}\left(K, \mathscr{G}_{a}\right)\right) \rightarrow \pi_{1}^{K}(\mathscr{G}) \stackrel{\pi_{1}(\omega)}{\rightarrow} \pi_{1}(\mathscr{G}) \stackrel{\partial}{\rightarrow} \pi_{0}\left(\operatorname{hom}(K, \mathscr{G})_{a, \rho}\right) \rightarrow 1 .
\end{aligned}
$$

Proof The proof is analogous to the proof of Theorem 7, with $\mathscr{G}^{K}$ replaced by $\mathscr{G}_{\rho}^{K}$ and $\operatorname{hom}\left(K, \mathscr{G}_{a}\right)$ replaced by $\operatorname{hom}\left(K, \mathscr{G}_{a, \rho}\right.$. We use that $\pi_{0}\left(\mathscr{G}_{\rho}^{K}\right)=1, \pi_{n}\left(\mathscr{G}^{K}\right)=$ $\pi_{n}\left(\mathscr{G}_{\rho}^{K}\right)$ and $\pi_{n}\left(\operatorname{hom}\left(K, \mathscr{G}_{a, \rho}\right)=\pi_{n}\left(\operatorname{hom}\left(K, \mathscr{G}_{a}\right)\right)\right.$ for any $n \geq 1$.

\section{Obstruction to presentability of proper regular Lie groupoids}

\subsection{Normal representation of a Lie groupoid}

Recall that a Lie groupoid is a topological groupoid $\mathscr{G}$ such that both $\mathscr{G}_{0}$ and $\mathscr{G}_{1}$ are smooth manifolds and all the structure maps are smooth, with the source map being a submersion. We also assume that $\mathscr{G}_{0}$ and the fibers of the source map are Hausdorff, but in general $\mathscr{G}_{1}$ may not be Hausdorff. Of course, we consider the smooth homomorphisms between Lie groupoids, smooth actions of Lie groupoids and smooth principal bundles. If $\mathscr{G}$ and $\mathscr{H}$ are Lie groupoids, then a smooth principal $\mathscr{G}$-bundle over $\mathscr{H}$ is a principal $\mathscr{G}$-bundle $P$ over $\mathscr{H}$ such that $P$ is a smooth manifold, all the structure maps of $P$ are smooth, the projection $P \rightarrow \mathscr{H}_{0}$ is a surjective submersion and the map $P \times \mathscr{G}_{0} \mathscr{G}_{1} \rightarrow P \times \mathscr{H}_{0} P,(p, g) \mapsto(p, p g)$, is a diffeomorphism. The (smooth) isomorphism classes of such smooth principal bundles are morphisms in the (smooth) Morita category of Lie groupoids (for details and examples, see [16,17]). Smooth manifolds and Lie groups are examples of Lie groupoids, as are the action groupoids of smooth actions of Lie groupoids. The isotropy groups of a Lie groupoid are Lie groups.

Recall that a Lie groupoid $\mathscr{G}$ is proper if it is Hausdorff and the map (s, t): $\mathscr{G}_{1} \rightarrow$ $\mathscr{G}_{0} \times \mathscr{G}_{0}$ is proper. A Lie groupoid $\mathscr{G}$ is regular if all its isotropy groups have the same dimension. A foliation Lie groupoid is a Lie groupoid with discrete isotropy groups. All these notions are stable under (smooth) Morita equivalence (see [16,17,21]). A Lie groupoid $\mathscr{G}$ is étale if its source map is a local diffeomorphism. A Lie groupoid is a foliation groupoid if and only if it is Morita equivalent to an étale Lie groupoid. Orbifolds can be viewed as proper foliation groupoids [14].

Let $\mathscr{G}$ be a Lie groupoid. For any $x \in \mathscr{G}_{0}$ we denote by $\mathcal{O}_{x}(\mathscr{G})=\mathscr{G} x$ the orbit of $\mathscr{G}$ through $x$. The orbit is an immersed submanifold of $\mathscr{G}_{0}$, with the smooth structure given by the principal $\mathscr{G}_{x}$-bundle $\mathrm{t}: \mathscr{G}(x,-) \rightarrow \mathcal{O}_{x}(\mathscr{G})$. The normal space of $\mathscr{G}$ at $x$ is the quotient $\mathrm{N}_{x} \mathscr{G}=\mathrm{T}_{x} \mathscr{G}_{0} / \mathrm{T}_{x} \mathcal{O}_{x}(\mathscr{G})$ of the tangent space of $\mathscr{G}_{0}$ and the tangent space of the orbit at $x$. The union $\mathrm{N} \mathscr{G}$ of all normal spaces $\mathrm{N}_{x} \mathscr{G}, x \in \mathscr{G}_{0}$, is a topological family of vector spaces which is locally trivial along an orbit, but not locally over $\mathscr{G}_{0}$ in general, as dimension of $\mathrm{N}_{x} \mathscr{G}$ may vary. There is a continuous linear left $\mathscr{G}$ action $v$ on $\mathrm{N}_{x} \mathscr{G}$ along the natural projection to $\mathscr{G}_{0}$. For any $g \in \mathscr{G}\left(x, x^{\prime}\right)$, the linear isomorphism

$$
v(g,-): \mathrm{N}_{x} \mathscr{G} \rightarrow \mathrm{N}_{x^{\prime}} \mathscr{G}
$$


is determined by the equality $v\left(g,\left[\left(d_{g} \mathrm{~s}\right)(v)\right]\right)=\left[\left(d_{g} \mathrm{t}\right)(v)\right]$ for any $v \in \mathrm{T}_{g} \mathscr{G}_{1}$ (for details, see [5]). This action restricts to a Lie group representation

$$
v_{x}: \mathscr{G}_{x} \rightarrow \mathrm{GL}\left(\mathrm{N}_{x} \mathscr{G}\right)
$$

and the kernel

$$
\mathrm{K}_{x}(\mathscr{G})=\operatorname{Ker}\left(v_{x}\right)
$$

is a closed Lie subgroup of $\mathscr{G}_{x}$. The union $\mathrm{K}(\mathscr{G})$ of $\mathrm{K}_{x}(\mathscr{G}), x \in \mathscr{G}_{0}$, is a topological family of Lie groups, a subfamily of the union $\mathrm{I}(\mathscr{G})$ of isotropy groups of $\mathscr{G}$, which is also a topological family of Lie groups. The elements of $\mathrm{K}(\mathscr{G})$ are called the ineffective arrows of $\mathscr{G}$. Note that $\mathrm{K}(\mathscr{G})$ is normal in $\mathscr{G}$, i.e. for any $g \in \mathscr{G}\left(x, x^{\prime}\right)$ we have $\operatorname{Ad}_{g}\left(\mathrm{~K}_{x}(\mathscr{G})\right)=\mathrm{K}_{x^{\prime}}(\mathscr{G})$, so we have a left (adjoint) $\mathscr{G}$-action on $\mathrm{K}(\mathscr{G})$ along the projection to $\mathscr{G}_{0}$. This follows from the equation $\operatorname{Ad}_{v(g,-)} \circ v_{x}=v_{x^{\prime}} \circ \operatorname{Ad}_{g}$.

It is important to note that the normal family $\mathrm{N} \mathscr{G}$, the normal action $v$, the isotropy family $\mathrm{I}(\mathscr{G})$ and the ineffective isotropy family $\mathrm{K}(\mathscr{G})$ all behave well with respect to (smooth) Morita morphisms. More precisely, let $P$ be a (smooth) principal $\mathscr{G}$-bundle over a Lie groupoid $\mathscr{H}$. Any $p \in P$ defines a linear map $d_{p} P: \mathrm{N}_{\pi(p)} \mathscr{H} \rightarrow \mathrm{N}_{\epsilon(p)} \mathscr{G}$, determined by the equality $d_{p} P\left(\left[\left(d_{p} \pi\right)(v)\right]\right)=\left[\left(d_{p} \epsilon\right)(v)\right]$ for any $v \in \mathrm{T}_{p} P$. One can check that $v_{\epsilon(p)}\left(P_{p}(h)\right) \circ d_{p} P=d_{p} P \circ v_{\pi(p)}(h)$ for any $h \in \mathscr{H}_{\pi(p)}$. If $P$ is a Morita equivalence, the maps $P_{p}$ and $d_{p} P$ are isomorphisms and $P_{p}\left(\mathrm{~K}_{\pi(p)}(\mathscr{H})\right)=$ $\mathrm{K}_{\epsilon(p)}(\mathscr{G})$.

If $\mathscr{G}$ is regular, then the connected components of the orbits of $\mathscr{G}$ define a regular foliation $\mathcal{F}(\mathscr{G})$ on $\mathscr{G}_{0}$, and $\mathrm{N} \mathscr{G}$ is a (locally trivial) smooth vector bundle on $\mathscr{G}_{0}$ equal to the normal bundle of the foliation $\mathcal{F}(\mathscr{G})$. The action $v$ of $\mathscr{G}$ on $\mathrm{N} \mathscr{G}$ is then a (smooth) representation of the Lie groupoid $\mathscr{G}$.

If $\mathscr{G}$ is regular and proper, then all the isotropy groups and all the ineffective isotropy groups are compact. However, this does not imply that the isotropy bundle I( $\mathscr{G})$ is a locally trivial bundle of Lie groups. It turns out, on the other hand, that this is true of $\mathrm{K}(\mathscr{G})$, at least if $\mathscr{G}$ is connected. In a different way, this was proved in [15] for the bundle $\mathrm{K}(\mathscr{G})^{\circ}$, which is the component of the trivial section in the bundle of $\mathrm{K}(\mathscr{G})$.

Proposition 10 If $\mathscr{G}$ is a connected proper regular Lie groupoid, then the ineffective isotropy bundle $\mathrm{K}(\mathscr{G})$ is a locally trivial bundle of Lie groups.

Proof This is a direct consequence of the local linearizability of proper regular Lie groupoids [4,21].

\subsection{Homotopy sequence of a proper regular Lie groupoid}

Proposition 11 Let $K$ be a compact Lie group, let $\mathscr{G}$ be a connected proper regular Lie groupoid with a basepoint a and let $\rho$ be a $K$-basegroup in $\mathscr{G}$ with image in $\mathrm{K}_{a}(\mathscr{G})$.

(i) Any element $\varphi \in \operatorname{hom}(K, \mathscr{G})_{\rho}$ has its image in $\mathrm{K}(\mathscr{G})$. If $\rho: K \rightarrow \mathrm{K}_{a}(\mathscr{G})$ is an isomorphism, then $\varphi: K \rightarrow \mathrm{K}_{\varphi(*)}(\mathscr{G})$ is also an isomorphism. 
(ii) The map $\omega: \operatorname{hom}(K, \mathscr{G})_{\rho} \rightarrow \mathscr{G}_{0}$ and the homomorphism $\omega: \mathscr{G}_{\rho}^{K} \rightarrow \mathscr{G}$ are Serre fibrations.

Proof (i) Recall that the space $\operatorname{hom}(K, \mathscr{G})_{\rho}$ is a minimal $\mathscr{G}$-invariant union of pathcomponents of the space $\operatorname{hom}(K, \mathscr{G})$. The bundle $\mathrm{K}(\mathscr{G})$ is normal in $\mathscr{G}$, and therefore it is sufficient to show that any $\varphi$, which is in the same path-component of the space $\operatorname{hom}(K, \mathscr{G})$ as $\rho$, has in fact image in $\mathrm{K}(\mathscr{G})$. In other words, if $\gamma$ is a path in $\operatorname{hom}(K, \mathscr{G})$ starting at $\rho$, we have to show that the image of $\gamma(t)$ is in $\mathrm{K}(\mathscr{G})$ for any $t \in[0,1]$. The path $\gamma$ gives us a path $\omega \circ \gamma$ in $\mathscr{G}_{0}$. Since $\mathscr{G}$ is proper and hence locally linearizable $[4,21]$, we may cover the image of $\omega \circ \gamma$ with finitely many open subsets of $\mathscr{G}_{0}$ on which the groupoid $\mathscr{G}$ is linearizable. We may therefore assume, without loss of generality, that the image of $\omega \circ \gamma$ lies in an open subset $U$ of $\mathscr{G}_{0}$ such that the restriction of $\mathscr{G}$ to $U$ is linearizable. Explicitly, this means that $\left.\mathscr{G}\right|_{U}$ is isomorphic to the restriction of the action groupoid

$$
\mathcal{N}_{\mathcal{O}}(\mathscr{G})=\left.\mathscr{G}\right|_{\mathcal{O}_{a}} \ltimes\left(\mathrm{s}^{-1}(a) \times \mathscr{G}_{a} \mathrm{~N}_{a}(\mathscr{G})\right)
$$

to an open subset $V$ of s ${ }^{-1}(a) \times \mathscr{G}_{a} \mathrm{~N}_{a}(\mathscr{G})$, where $\left.\mathscr{G}\right|_{\mathcal{O}_{a}}$ is the transitive groupoid obtained as the restriction of $\mathscr{G}$ to the orbit $\mathcal{O}_{a}$ of $\mathscr{G}$ through $a$. The isomorphism $\phi$ from $\mathscr{G}_{U}$ to $\left.\mathcal{N}_{\mathcal{O}}(\mathscr{G})\right|_{V}$ is the canonical one when restricted to the orbit $\mathcal{O}_{a}$.

The quotient map $q: \mathrm{s}^{-1}(a) \times \mathrm{N}_{a}(\mathscr{G}) \rightarrow \mathrm{s}^{-1}(a) \times \mathscr{G}_{a} \mathrm{~N}_{a}(\mathscr{G})$ is a principal $\mathscr{G}_{a^{-}}$ bundle, so we can lift the path $\phi \circ \omega \circ \gamma$ to a path $\tilde{\gamma}$ in $\mathrm{s}^{-1}(a) \times \mathrm{N}_{a}(\mathscr{G})$. We write $\tilde{\gamma}(t)=\left(g_{t}, v_{t}\right)$ and put $\alpha(t)=\operatorname{Ad}_{g_{t}^{-1}} \circ \phi_{*}(\gamma(t))$. Note that $\alpha(t)$ is a homomorphism from $K$ to the isotropy group of $\mathcal{N}_{\mathcal{O}}(\mathscr{G})$ at $q\left(1_{a}, v_{t}\right)$, which is a subgroup of $\mathscr{G}_{a}$. Since $\alpha(t)$ depends continuously on $t$, there exist $h_{t} \in \mathscr{G}_{a}$ such that $\alpha(t)=\operatorname{Ad}_{h_{t}} \circ \alpha(0)$ (see [3, Lemma 38.1]). It follows that

$$
\alpha(t)=\operatorname{Ad}_{h_{t}} \circ \operatorname{Ad}_{g_{0}^{-1}} \circ \phi_{*}(\gamma(0))=\operatorname{Ad}_{h_{t} g_{0}^{-1}} \circ \phi_{*}(\rho)
$$

and this implies the first part of the statement. The second part of the statement follows along the same lines.

(ii) We know that $\mathrm{K}(\mathscr{G})$ is a locally trivial bundle of Lie groups over $\mathscr{G}_{0}$, so $\omega$ : $\operatorname{hom}(K, \mathrm{~K}(\mathscr{G})) \rightarrow \mathscr{G}_{0}$ is also a locally trivial bundle and therefore a Serre fibration. The same is true for the restriction of $\omega$ to a $\mathscr{G}$-invariant union of path-connected components of $\operatorname{hom}(K, \mathrm{~K}(\mathscr{G}))$. On the other hand, the space $\operatorname{hom}(K, \mathrm{~K}(\mathscr{G}))$ is a union of path-connected components of $\operatorname{hom}(K, \mathscr{G})$ by (i).

As a consequence of Proposition 11, we can apply Theorem 9, to obtain a long exact sequence of homotopy groups for any proper regular $K$-pointed Lie groupoid $(\mathscr{G}, a, \rho)$ such that $\rho(K) \subset \mathrm{K}_{a}(\mathscr{G})$. However, if $\rho$ is an isomorphism onto $\mathrm{K}_{a}(\mathscr{G})$, the long exact sequence could be written in a simpler form:

Theorem 12 Let $K$ be a compact Lie group, let $\mathscr{G}$ be a connected proper regular Lie groupoid with basepoint $a$ and let $\rho: K \rightarrow \mathrm{K}_{a}(\mathscr{G})$ be an isomorphism. We have a long exact sequence 


$$
\begin{aligned}
& \cdots \rightarrow \pi_{n}\left(\operatorname{Aut}\left(\mathrm{K}_{a}(\mathscr{G})\right)\right) \rightarrow \pi_{n}^{K}(\mathscr{G}) \stackrel{\pi_{n}(\omega)}{\rightarrow} \pi_{n}(\mathscr{G}) \stackrel{\partial}{\rightarrow} \pi_{n-1}\left(\operatorname{Aut}\left(\mathrm{K}_{a}(\mathscr{G})\right)\right) \rightarrow \cdots \\
& \cdots \rightarrow \pi_{1}\left(\operatorname{Aut}\left(\mathrm{K}_{a}(\mathscr{G})\right)\right) \rightarrow \pi_{1}^{K}(\mathscr{G}) \stackrel{\pi_{1}(\omega)}{\rightarrow} \pi_{1}(\mathscr{G}) \stackrel{\partial}{\rightarrow} \pi_{0}\left(\operatorname{Aut}\left(\mathrm{K}_{a}(\mathscr{G})\right)\right)
\end{aligned}
$$

which is natural with respect to Morita equivalences.

Remark Here we used the isomorphism $\rho$ to identify the space of isomorphisms from $K$ to $\mathrm{K}_{a}(\mathscr{G})$ with $\operatorname{Aut}\left(\mathrm{K}_{a}(\mathscr{G})\right)$. Note that $\pi_{n}\left(\operatorname{Aut}\left(\mathrm{K}_{a}(\mathscr{G})\right)\right.$, id $)=\pi_{n}\left(\operatorname{Aut}\left(\mathrm{K}_{a}(\mathscr{G})\right)\right)=$ $\pi_{n}\left(\operatorname{Inn}\left(\mathrm{K}_{a}(\mathscr{G})\right)\right)=\pi_{n}\left(\mathrm{~K}_{a}(\mathscr{G}) / \mathrm{C}\left(\mathrm{K}_{a}(\mathscr{G})\right)\right)$ for $n \geq 1$. The sequence ends with the homomorphism

$$
\partial: \pi_{1}(\mathscr{G}, a) \rightarrow \pi_{0}\left(\operatorname{Aut}\left(\mathrm{K}_{a}(\mathscr{G})\right)\right)
$$

which we call the monodromy map. This homomorphism does not depend on the choice of $\rho$, thus it is a Morita invariant of $(\mathscr{G}, a)$. To understand the monodromy map geometrically, one should represent an element of $\pi_{1}(\mathscr{G}, a)$ by a $\mathscr{G}$-cocycle on $I$, i.e. a series of paths in $\mathscr{G}_{0}$ connected by arrows of $\mathscr{G}$ (see [17]). Then the monodromy is obtained by a combination of lifts along the paths and adjoint actions of the connecting arrows.

Proof This follows from Theorem 9 and Proposition 11.

Example 13 Let $\mathscr{G}$ be a connected proper étale Lie groupoid. Any such groupoid is regular, has discrete orbits and discrete isotropy groups. We have the associated effect homomorphism $\mathscr{G} \rightarrow \operatorname{Eff}(\mathscr{G})[16]$ and $\mathrm{K}(\mathscr{G})$ is exactly its kernel. By Theorem 12 we have the long exact sequence

$$
\cdots \rightarrow \pi_{n}\left(\operatorname{Aut}\left(\mathrm{K}_{a}(\mathscr{G})\right)\right) \rightarrow \pi_{n}^{K}(\mathscr{G}) \stackrel{\pi_{n}(\omega)}{\rightarrow} \pi_{n}(\mathscr{G}) \stackrel{\partial}{\rightarrow} \pi_{n-1}\left(\operatorname{Aut}\left(\mathrm{K}_{a}(\mathscr{G})\right)\right) \rightarrow \cdots
$$

for a choice of a point $a \in \mathscr{G}_{0}$ and an isomorphism of Lie groups $\rho: K \rightarrow \mathrm{K}_{a}(\mathscr{G})$. Since the isotropy groups of $\mathscr{G}$ are discrete, the space $\operatorname{Aut}\left(\mathrm{K}_{a}(\mathscr{G})\right)$ is discrete as well. The long exact sequence thus implies that

$$
\pi_{n}(\omega): \pi_{n}^{K}(\mathscr{G}) \rightarrow \pi_{n}(\mathscr{G})
$$

is an isomorphism for any $n \geq 2$ and that we have an exact sequence

$$
1 \rightarrow \pi_{1}^{K}(\mathscr{G}) \stackrel{\pi_{1}(\omega)}{\rightarrow} \pi_{1}(\mathscr{G}) \stackrel{\partial}{\rightarrow} \operatorname{Aut}\left(\mathrm{K}_{a}(\mathscr{G})\right)
$$

Example 14 Let $G$ be a connected compact Lie group acting on a connected manifold $M$, and assume that this action is regular, i.e. that the isotropy groups of this action have constant dimension. Therefore, the associated action groupoid $G \ltimes M$ is regular and proper. The isotropy group $G_{x}=(G \ltimes M)_{x}$ is a subgroup of $G$, for any $x \in M$. Choose a basepoint $x \in M$, write $\mathrm{K}_{x}=\mathrm{K}_{x}(G \ltimes M)$ and let $\rho: K \rightarrow \mathrm{K}_{x}$ be an isomorphism of Lie groups. Note that $\mathrm{K}_{x}$ is a normal subgroup of $G_{x}$ and in particular a subgroup of $G$. By Theorem 12 we have the long exact sequence 


$$
\cdots \rightarrow \pi_{n}\left(\operatorname{Aut}\left(\mathrm{K}_{x}\right)\right) \rightarrow \pi_{n}^{K}(G \ltimes M) \stackrel{\pi_{n}(\omega)}{\rightarrow} \pi_{n}(G \ltimes M) \stackrel{\partial}{\rightarrow} \pi_{n-1}\left(\operatorname{Aut}\left(\mathrm{K}_{x}\right)\right) \rightarrow \cdots
$$

This sequence ends with the monodromy map

$$
\partial: \pi_{1}(G \ltimes M, x) \rightarrow \pi_{0}\left(\operatorname{Aut}\left(\mathrm{K}_{x}\right)\right) .
$$

Note that, by construction and by [3, Lemma 38.1], we have $\partial\left(\pi_{1}(G \ltimes M, x)\right) \subset$ $\pi_{0}\left(\mathrm{~W}_{G}\left(\mathrm{~K}_{x}\right)\right)$, where $\mathrm{W}_{G}\left(\mathrm{~K}_{x}\right)$ is the subgroup of Aut $\left(\mathrm{K}_{x}\right)$ consisting of all automorphisms of $\mathrm{K}_{x}$ which are the restriction of an inner automorphism of $G$. The group $\mathrm{W}_{G}\left(\mathrm{~K}_{x}\right)$ is isomorphic to the quotient $\mathrm{N}_{G}\left(\mathrm{~K}_{x}\right) / \mathrm{C}_{G}\left(\mathrm{~K}_{x}\right)$ of the normalizer of $\mathrm{K}_{x}$ in $G$ and the centralizer of $\mathrm{K}_{x}$ in $G$.

We may choose a faithful unitary representation of the compact Lie group $G$. This representation restricts to a faithful unitary representation $r: \mathrm{K}_{x} \rightarrow \mathrm{U}(m)$ of $\mathrm{K}_{x}$. We write $\mathrm{W}_{r}\left(\mathrm{~K}_{x}\right)$ for the subgroup of $\operatorname{Aut}\left(\mathrm{K}_{x}\right)$ which is identified with the group $\mathrm{W}_{\mathrm{U}(m)}\left(r\left(\mathrm{~K}_{x}\right)\right)$ by $r$. Note that $\mathrm{W}_{G}\left(\mathrm{~K}_{x}\right) \subset \mathrm{W}_{r}\left(\mathrm{~K}_{x}\right)$, so $\partial\left(\pi_{1}(G \ltimes M, x)\right) \subset$ $\pi_{0}\left(\mathrm{~W}_{G}\left(\mathrm{~K}_{x}\right)\right)$ implies

$$
\partial\left(\pi_{1}(G \ltimes M, x)\right) \subset \pi_{0}\left(\mathrm{~W}_{r}\left(\mathrm{~K}_{x}\right)\right) .
$$

\subsection{Obstruction to presentability}

For a given proper Lie groupoid $\mathscr{G}$ it is a natural question to ask whether it is presentable as a global quotient, or more precisely, whether it is Morita equivalent to the action groupoid associated to an action of a compact connected Lie group. Any effective étale proper Lie groupoid (such groupoids represent effective orbifolds) is Morita equivalent to the action groupoid of an action of a compact connected Lie group on a manifold (see e.g. [16]). On the other hand, there exist particular examples of connected proper regular Lie groupoids which are not presentable in this way, while it is not yet known whether all proper étale Lie groupoids are presentable.

Let $\mathscr{G}$ be a connected proper regular Lie groupoid with basepoint $a \in \mathscr{G}_{0}$. The associated monodromy map

$$
\partial: \pi_{1}(\mathscr{G}, a) \rightarrow \pi_{0}\left(\operatorname{Aut}\left(\mathrm{K}_{a}(\mathscr{G})\right)\right)
$$

is natural with respect to Morita equivalences. In particular, if $\mathscr{G}$ is Morita equivalent to the action groupoid $G \ltimes M$ of an action of a connected compact Lie group $G$ on a connected manifold $M$, then the groupoid $G \ltimes M$ is regular and we have the commutative diagram

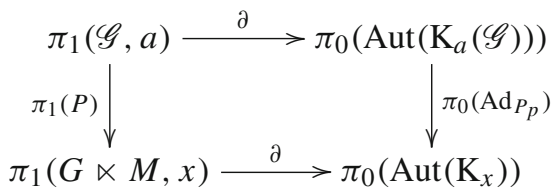


where $P$ is a Morita equivalence from $\mathscr{G}$ to $G \ltimes M, p$ is a point in $P$ such that $a=\pi(p), x=\epsilon(p)$ and $\mathrm{K}_{x}=\mathrm{K}_{x}(G \ltimes M) \subset G_{x} \subset G$. By Example 14 we know that $\partial\left(\pi_{1}(G \ltimes M, x)\right) \subset \pi_{0}\left(\mathrm{~W}_{G}\left(\mathrm{~K}_{x}\right)\right)$, and we obtain the following obstruction to presentability:

Theorem 15 Let $\mathscr{G}$ be a connected proper regular Lie groupoid with basepoint a $\in$ $\mathscr{G}_{0}$. If $\mathscr{G}$ is Morita equivalent to the action groupoid associated to an action of a connected compact Lie group on a smooth manifold, then there exists a faithful unitary representation $r: \mathrm{K}_{a}(\mathscr{G}) \rightarrow \mathrm{U}(m)$ such that $\partial\left(\pi_{1}(\mathscr{G}, a)\right) \subset \pi_{0}\left(\mathrm{~W}_{r}\left(\mathrm{~K}_{a}(\mathscr{G})\right)\right)$.

Remark Observe that the condition $\partial\left(\pi_{1}(\mathscr{G}, a)\right) \subset \pi_{0}\left(\mathrm{~W}_{r}\left(\mathrm{~K}_{a}(\mathscr{G})\right)\right)$ is satisfied if and only if the character of the representation $r$ is fixed under precomposition with any automorphism representing a class in the image of the monodromy map $\partial$ : $\pi_{1}(\mathscr{G}, a) \rightarrow \pi_{0}\left(\operatorname{Aut}\left(\mathrm{K}_{a}(\mathscr{G})\right)\right)$.

Example 16 Let $G$ be a Lie group, let $M$ be a connected manifold with basepoint $a$ and let $R: \pi_{1}(M, a) \rightarrow \operatorname{Aut}(G)$ be a homomorphism. Choose a universal covering space $q: \tilde{M} \rightarrow M$ and basepoint $\tilde{a} \in q^{-1}(a)$. We have a natural right action of $\pi_{1}(M, a)$ on $\tilde{M}$ by deck transformations. Associated to $R$ we have a Lie groupoid $\mathscr{B}$ over $M$ with space of arrows

$$
\mathscr{B}_{1}=\tilde{M} \times_{\pi_{1}(M, a)} G
$$

which is the space of orbits of the diagonal $\pi_{1}(M, a)$-action on $\tilde{M} \times G$. With source and target map of $\mathscr{B}$ both being equal to the natural projection to $M$, the Lie groupoid $\mathscr{B}$ is in fact a locally trivial bundle of Lie groups over $M$. The long exact sequence of the Serre fibration $M \rightarrow \mathscr{B}$ gives us the short exact sequence

$$
1 \rightarrow \pi_{n}(M) \rightarrow \pi_{n}(\mathscr{B}) \rightarrow \pi_{n-1}(G) \rightarrow 1
$$

for any $n \geq 1$. In particular, the group $\pi_{1}(M, a)$ is a subgroup of $\pi_{1}(\mathscr{B}, a)$.

The Lie groupoid $\mathscr{B}$ is regular with $\mathrm{I}(\mathscr{B})=\mathrm{K}(\mathscr{B})=\mathscr{B}$. Since $\mathscr{B}$ is a locally trivial bundle of Lie groups, it follows that $\omega: \operatorname{hom}(K, \mathscr{B}) \rightarrow M$ is also a locally trivial bundle and hence a Serre fibration, so $\omega: \mathscr{B}^{K} \rightarrow \mathscr{B}$ is a Serre fibration, for any Lie group $K$. For any choice of a $K$-basegroup $\rho$ in $\mathscr{B}$ we therefore have the long exact sequence

$$
\cdots \rightarrow \pi_{n}(\operatorname{hom}(K, \mathscr{B})) \rightarrow \pi_{n}^{K}(\mathscr{B}) \stackrel{\pi_{n}(\omega)}{\rightarrow} \pi_{n}(\mathscr{B}) \stackrel{\partial}{\rightarrow} \pi_{n-1}(\operatorname{hom}(K, \mathscr{B})) \rightarrow \cdots
$$

In particular, if $G$ is compact, $K=G$ and $\rho: G \rightarrow \mathscr{B}_{a}$ is the natural isomorphism of Lie groups, we get the long exact sequence

$$
\cdots \rightarrow \pi_{n}\left(\operatorname{Aut}\left(\mathscr{B}_{a}\right)\right) \rightarrow \pi_{n}^{G}(\mathscr{B}) \stackrel{\pi_{n}(\omega)}{\rightarrow} \pi_{n}(\mathscr{B}) \stackrel{\partial}{\rightarrow} \pi_{n-1}\left(\operatorname{Aut}\left(\mathscr{B}_{a}\right)\right) \rightarrow \cdots
$$

At the end of this sequence we have the map $\partial: \pi_{1}(\mathscr{B}, a) \rightarrow \pi_{0}\left(\operatorname{Aut}\left(\mathscr{B}_{a}\right)\right)$. The restriction of this homomorphism to $\pi_{1}(M, a)$ is exactly the composition of $R$ with the natural quotient map $\operatorname{Aut}(G) \rightarrow \pi_{0}(\operatorname{Aut}(G)) \cong \pi_{0}\left(\operatorname{Aut}\left(\mathscr{B}_{a}\right)\right)$. 
If the Lie groupoid $\mathscr{B}$ is presentable as a global quotient, the previous theorem implies that there exists a faithful representation $r: G \rightarrow U(m)$ such that $\partial\left(\pi_{1}(\mathscr{B}, a)\right) \subset \pi_{0}\left(\mathrm{~W}_{r}(G)\right)$. In particular, this implies

$$
R\left(\pi_{1}(M, a)\right) \subset \mathrm{W}_{r}(G) .
$$

In other words, if we choose the homomorphism $R$ so that this condition is not satisfied for any faithful representation $r$, then $\mathscr{B}$ is not presentable. Finding out if such $R$ exists is not too difficult when we know the group $G$ well enough, e.g. if we explicitly know all of its irreducible characters and all characters of its faithful representations.

For example, it is easy to find such $R$ for the case where $G$ is the $n$-torus $T^{n}, n \geq 2$. Let $M=S^{1}$ and $G=T^{n}=(\mathrm{U}(1))^{n} \subset \mathbb{C}^{n}$. Then $\pi_{1}\left(S^{1}, a\right)=\mathbb{Z}$ and a homomorphism $R: \pi_{1}\left(S^{1}, a\right) \rightarrow \operatorname{Aut}\left(T^{n}\right)$ is determined by a Lie group automorphism $R(1): T^{n} \rightarrow T^{n}$. Such an automorphism $R(1)$ is given by an integer matrix $A$ of dimension $n \times n$ with $\operatorname{det} A= \pm 1$, by

$$
R(1)\left(z_{1}, z_{2}, \ldots, z_{n}\right)=\left(z_{1}^{A_{11}} z_{2}^{A_{12}} \cdots z_{n}^{A_{1 n}}, \ldots, z_{1}^{A_{n 1}} z_{2}^{A_{n 2}} \cdots z_{n}^{A_{n n}}\right)
$$

for any $z_{1}, z_{2} \ldots, z_{n} \in \mathrm{U}(1) \subset \mathbb{C}$. Note that $A$ is the derivative of $R(1)$ at the identity element, so $A$ is in fact the Lie algebra automorphism associated to the Lie group automorphism $R(1)$. Any representation of $T^{n}$ is equivalent to one of the form $r=\left(\chi_{1}, \ldots, \chi_{m}\right): T^{n} \rightarrow(\mathrm{U}(1))^{m} \subset \mathrm{U}(m)$, where $\chi_{1}, \ldots, \chi_{m}: T^{n} \rightarrow \mathrm{U}(1)$ are Lie group homomorphisms. Explicitly, we have $\chi_{i}\left(z_{1}, z_{2}, \ldots, z_{n}\right)=z_{1}^{C_{i 1}} z_{2}^{C_{i 2}} \cdots z_{n}^{C_{i n}}$ for an integer matrix $C$ of dimension $m \times n$. Write $\chi=\chi_{1}+\cdots+\chi_{m}$ for the character of $r$. If $\chi \circ R(1)=\chi$, then

$$
\chi_{1} \circ R(1)+\cdots+\chi_{m} \circ R(1)=\chi_{1}+\cdots+\chi_{m}
$$

and hence the sequence of irreducible characters $\left(\chi_{1} \circ R(1), \ldots, \chi_{m} \circ R(1)\right)$ is just a permutation of the sequence $\left(\chi_{1}, \ldots, \chi_{m}\right)$. In particular, there exists $k \geq 1$ such that $r \circ R(1)^{k}=r$. If $r$ is faithful, this yields $R(1)^{k}=$ id and therefore $A^{k}=I$. Hence, if we choose $A$ to be any integer matrix of dimension $n \times n$ with $\operatorname{det} A= \pm 1$ and of infinite multiplicative order, then the associated Lie groupoid $\mathscr{B}$ over $S^{1}$ is not presentable. (This example of non-presentable proper Lie groupoid is known for $n=2$ with the matrix $A$ being upper triangular, see e.g. $[11,20])$.

\section{References}

1. Carchedi, D.: Compactly generated stacks: a Cartesian closed theory of topological stacks. Adv. Math. 229(6), 3339-3397 (2012)

2. Chen, W.: On a notion of maps between orbifolds. II. Homotopy and CW-complex. Commun. Contemp. Math. 8(6), 763-821 (2006)

3. Conner, P.E., Floyd, E.E.: Differentiable Periodic Maps. Springer, Berlin (1964)

4. Crainic, M., Struchiner, I.: On the linearization theorem for proper Lie groupoids. arXiv:1103.5245v1 (2011)

5. Evens, S., Lu, J.-H., Weinstein, A.: Transverse measures, the modular class and a cohomology pairing for Lie algebroids. Q. J. Math. Oxf. Ser. (2) 50(200), 417-436 (1999) 
6. Haefliger, A.: Homotopy and integrability. In: Manifolds-Amsterdam 1970 (Proceedings of the Nuffic Summer School). Lecture Notes in Mathematics, vol. 197, pp. 133-163 (1971)

7. Haefliger, A.: Groupoïdes d'holonomie et classifiants. Transversal structure of foliations (Toulouse, 1982). Astérisque 116, 70-97 (1984)

8. Haefliger, A.: On the space of morphisms between étale groupoids. A celebration of the mathematical legacy of Raoul Bott. In: CRM Proceedings and Lecture Notes, vol. 50, pp. 139-150. American Mathematical Society, Providence (2010)

9. Henriques, A., Gepner, D.: Homotopy theory of orbispaces. arXiv:math/0701916v1 (2007)

10. Jelenc, B.: Serre fibrations in the Morita category of topological groupoids. Topol. Appl. 160(1), 9-23 (2013)

11. Lück, W., Oliver, B.: The completion theorem in $K$-theory for proper actions of a discrete group. Topology 40(3), 585-616 (2001)

12. Mackenzie, K.C.H.: General Theory of Lie Groupoids and Lie Algebroids. Cambridge University Press, Cambridge (2005)

13. Moerdijk, I.: Classifying Spaces and Classifying Topoi. Springer, Berlin (1995)

14. Moerdijk, I.: Orbifolds as groupoids: an introduction. Orbifolds in mathematics and Physics (Madison 2001). Contemp. Math. 310, 205-222 (2002)

15. Moerdijk, I.: Lie groupoids, gerbes, and non-abelian cohomology.K-Theory 28(3), 207-258 (2003)

16. Moerdijk, I., Mrčun, J.: Introduction to Foliations and Lie Groupoids. Cambridge University Press, Cambridge (2003)

17. Moerdijk, I., Mrčun, J.: Lie groupoids, sheaves and cohomology. Poisson geometry, deformation quantisation and group representations. Lond. Math. Soc. Lect. Note Ser. 323, 145-272 (2005)

18. Mrčun, J.: Stability and invariants of Hilsum-Skandalis maps. PhD thesis, Utrecht University. arXiv:math/0506484v1 (1996)

19. Noohi, B.: Fibrations of topological stacks. arXiv:1010.1748v1 (2010)

20. Trentinaglia, G.: On the role of effective representations of Lie groupoids. Adv. Math. 225(2), 826-858 (2010)

21. Weinstein, A.: Linearization of regular proper groupoids. J. Inst. Math. Jussieu 1(3), 493-511 (2002) 